\title{
Impact of early anti-TNF use on clinical outcomes in Crohn's disease: a nationwide population-based study
}

\author{
Yoon Suk Jung ${ }^{1,}$, Minkyung Han ${ }^{2,}$, , Sohee Park ${ }^{3}$, and Jae Hee Cheon ${ }^{4}$
}

\begin{abstract}
${ }^{1}$ Division of Gastroenterology, Department of Internal Medicine, Kangbuk Samsung Hospital, Sungkyunkwan University School of Medicine, Seoul; ${ }^{2}$ Biostatistics Collaboration Unit, Department of Biomedical Systems Informatics, Yonsei University College of Medicine, Seoul; ${ }^{3}$ Department of Biostatistics, Yonsei University Graduate School of Public Health, Seoul; ${ }^{4}$ Department of Internal Medicine and Institute of Gastroenterology, Yonsei University College of Medicine, Seoul, Korea
\end{abstract}

Received: January 1, 2020

Revised : March 6, 2020

Accepted: March 17, 2020

\section{Correspondence to}

Jae Hee Cheon, M.D.

Department of Internal Medicine, Yonsei University College of Medicine, 50-1 Yonsei-ro, Seodaemun-gu, Seoul 03722, Korea Tel: +82-2-2228-1990

Fax: +82-2-393-6884

E-mail: GENIUSHEE@yuhs.ac https://orcid.org/0000-00022282-8904

${ }^{*}$ These authors contributed equally to this work.
Background/Aims: The optimal timing for initiation of anti-tumor necrosis factor (TNF) therapy in Crohn's disease (CD) is still debated. Little is known about the clinical outcomes of early versus late administration of anti-TNF agents, especially in Asian CD patients. We aimed to evaluate the impact of early anti-TNF therapy on clinical outcomes in Korean CD patients.

Methods: Using the Korean National Health Insurance Claims database, we collected data on patients diagnosed with CD who received anti-TNF therapy for more than 6 months between 2010 and 2016. Early initiation of anti-TNF therapy was defined as those starting infliximab or adalimumab therapy within 1 year of diagnosis. The following outcomes were assessed using a Cox proportional hazard model: abdominal surgery, CD-related emergency room (ER) visit, CD-related hospitalization, and new corticosteroid use.

Results: Among 1,207 patients, 609 were early initiators of anti-TNF. Late anti-TNF initiation ( $>1$ year after diagnosis) was associated with increased risk of surgery (adjusted hazard ratio [aHR], 1.64; 95\% confidence interval [CI], 1.05 to 2.55) and tended to be associated with increased risk of ER visit (aHR, 1.38; 95\% CI, 0.99 to 1.94). However, there were no significant differences in the risk of hospitalization and corticosteroid use between early and late initiators.

Conclusions: Early anti-TNF therapy among Korean CD patients within 1 year of diagnosis was associated with better clinical outcomes than late therapy, such as lower surgery and ER visit rates. Our results suggest that aggressive medical intervention in the early stages of CD may potentially change the course of this disease.

Keywords: Crohn disease; Immunotherapy; Tumor necrosis factor-alpha

\section{INTRODUCTION}

Crohn's disease (CD) is a relapsing and remitting inflammatory bowel disease (IBD) that leads to bowel damage and impaired gut function [1]. It is a chronic and incurable disease that requires life-long medical treatment. The medical management of CD has dramatically changed over the past few decades with the advent of biological agents targeting tumor necrosis factor-alpha (TNF- $\alpha$ ), including infliximab and adalimumab [2]. Mucosal healing induced by these agents appears to bring about changes in the natural history of CD [3]. The ACCENT II trial (A Crohn's Disease Clinical Trial Evaluating Infliximab in a New Long-Term Treatment Reg- 
imen in Patients with Fistulizing Crohn's Disease) and CHARM (Crohn's Trial of the Fully Human Antibody Adalimumab for Remission Maintenance) studies revealed that infliximab and adalimumab maintenance therapy reduced hospitalization and surgeries in patients with CD $[4,5]$.

Although anti-TNF therapy is associated with better clinical outcomes, the use of anti-TNF agents is often postponed until after failure of other treatment modalities. A delay in effective medical therapy can lead to chronic and uncontrolled inflammation, resulting in irreversible complications such as stenosis and fistula. Indeed, previous studies have shown that use of immunomodulators (IMs) and/or biologics early in the course of the disease, known as top-down or accelerated step-up strategies, is superior to conventional step-up therapy in patients with $C D[3,6,7]$. In addition, several recent studies have compared early and late initiation of anti-TNF therapy in CD and have demonstrated better clinical outcomes in early initiators than in late initiators [8-11]. However, most previous studies on this topic have been conducted in Western countries. Relatively little is known about the effectiveness of early use of anti-TNF agents in Asian patients with CD, and it is questionable whether the results of studies involving Western patients can be extrapolated to Asian patients. The optimal timing of anti-TNF therapy initiation remains a challenging issue, especially in Asian patients with CD.

Therefore, we performed a nationwide populationbased study to assess the effect of early initiation of anti-TNF agents on clinical outcomes in Korean patients with $\mathrm{CD}$, by using a health claims administrative database. We compared clinical outcomes (including abdominal surgery, CD-related emergency room (ER) visits, hospitalization rates, and new corticosteroid use) between early versus late initiators of infliximab or adalimumab during maintenance therapy in a real-life clinical setting.

\section{METHODS}

\section{Study design and data source}

We conducted a retrospective population-based study using a database provided by the National Health Insurance Service (NHIS) in South Korea between 2008 and 2016. The Korean NHIS is a mandatory nationwide insurance system that covers the entire population of South Korea (approximately 51 million people). All information regarding health care utilization gathered by the NHIS is registered in a comprehensive database, which is operated by the Health Insurance Review and Assessment Service (HIRA). Medical institutions electronically submit all health care use information to ensure reimbursement, and this information is integrated into the HIRA database. The HIRA database covers a wide range of information on all patients, including demographic characteristics, ambulatory care history, prescriptions, procedures, and diagnostic codes defined by the International Classification of Diseases 1oth Revision (ICD-10) [12-14].

All identifiable personal information in the medical records was de-identified to comply with the Health Insurance Portability and Accountability Act privacy rule. In addition, as the information in the HIRA database is encrypted, the database does not contain personal identifiers. This study protocol was approved by the Severance Hospital Institutional Review Board (IRB), Yonsei University College of Medicine (IRB No. 4-2017-0927). Written informed consent by the patients was waived due to a retrospective nature of our study.

\section{Patient identification and definitions}

To ensure that only patients accurately diagnosed as having CD were analyzed, we only included patients with both appropriate diagnostic codes as well as CD-related drug prescriptions [12-14]. ICD-10 codes K50.0-50.9 were used to denote $\mathrm{CD}$. CD-related drug prescriptions were defined as prescriptions of 5-aminosalicylic acid (5-ASA) for 1 month or more, IMs (azathioprine or 6-mercaptopurine) at least once, and/or biologics at least once [1214]. To rule out the use of drugs for other autoimmune diseases, only prescriptions received from gastroenterology clinics were considered.

The date of CD registration in the HIRA database was regarded as the date of diagnosis. Considering that previous prevalent cases could confound the incidence rate, we used a washout period of 2 years; thus, we were able to analyze patients who were diagnosed with $\mathrm{CD}$ and received anti-TNF therapy between January 1, 2010 and December 31, 2016.

Because the effects of anti-TNF therapy on long-term outcomes could not be assessed with short-term treat- 
ment, patients were excluded from the analysis if they underwent anti-TNF therapy for less than 6 months. Patients with a history of abdominal surgery before starting anti-TNF therapy were also excluded from the analysis, as the efficacy of anti-TNF therapy can differ between patients with and those without a history of surgery.

Patients were stratified by the time of first dose of induction anti-TNF agent after the diagnosis. Early initiation of anti-TNF therapy was defined as starting infliximab or adalimumab therapy within 1 year of diagnosis, whereas late initiation of anti-TNF therapy was defined as starting infliximab or adalimumab therapy $>1$ year after diagnosis.

Because of the poor performance of claims codes for perianal diseases, we considered the surgical history of perianal diseases. Surgical history of perianal disease was defined as the presence of surgical procedure codes for anal fissure (Q2950), anal fistula (Q2974, Q2975, Q2976 Q2977, Q2978, and Q2979), or periproctal abscess (Q2881, Q2882, and Q2883) before starting biological therapy.

\section{Outcomes}

The primary objective of this study was to determine if early initiation of anti-TNF therapy affected the need for (1) abdominal surgery, (2) CD-related ER visits, (3) CD-related hospitalization, or (4) new corticosteroid use during anti-TNF maintenance therapy. Abdominal surgery was identified by using the procedural code for major surgery and defined by the presence of surgical procedure codes with bowel resection as follows: colectomy (Q1261, Q1262, Q2672, Q2673, Q2679, QA672, and QA673), total coloprotectomy (QA925), right or left hemicolectomy (Q2671 and QA671), resection of the small intestine (Q2650 and Q2651), and resection of intestine (Q2691, Q2761, and Q2771). Appendectomy (Q2861, Q2862, and Q2863) was not included in the definition of abdominal surgery. ER visits were defined as patient visits to the ER with $\mathrm{CD}$ as the primary diagnosis. Hospitalizations were defined as cases where the patient was admitted to the department of gastroenterology for 3 days or more. New corticosteroid use was defined as moderate- to highdose corticosteroid use ( $\geq 30 \mathrm{mg}$ prednisolone, $\geq 50 \mathrm{mg}$ methylprednisolone, or $\geq 200 \mathrm{mg}$ hydrocortisone) 2 months after the first prescription of anti-TNF therapy.

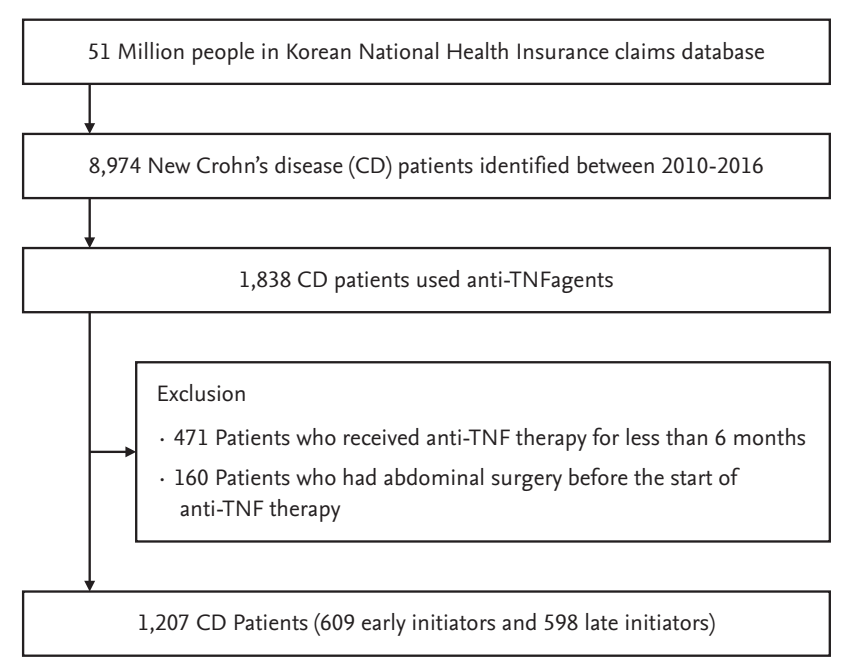

Figure 1. Flow diagram for patient selection. TNF, tumor necrosis factor.

\section{Statistical analysis}

Incidence rates for surgery, ER visits, hospitalizations, and the need for corticosteroids were calculated per 100 person-years, with 95\% confidence intervals (CIs) using a Poisson distribution. The crude risk of outcomes in early versus late initiators was compared by the Kaplan-Meier method and log-rank tests. We used Cox proportional hazards models to adjust for potential confounding variables. Baseline factors including sex, age, type of first anti-TNF agent (infliximab or adalimumab), region, hospital scale, and surgical history of perianal disease were adjusted as time-fixed covariates. The period of use of anti-TNF agents and the use of IMs were adjusted as time-dependent covariates. The results are presented as hazard ratios (HRs) with corresponding 95\% CIs. All analyses were performed using the SAS Enterprise Guide (SAS Institute Inc., Cary, NC, USA), and $p$ values $<0.05$ were considered statistically significant.

\section{RESULTS}

\section{Baseline patient demographics}

We identified 8,974 patients diagnosed with CD between 2010 and 2016, 1,838 of whom started their first anti-TNF agents during the study period. Of these 1,838 patients, 471 were excluded because they received anti-TNF therapy for a period of less than 6 months, and 160 were excluded because they underwent abdominal 
Abdominal surgery

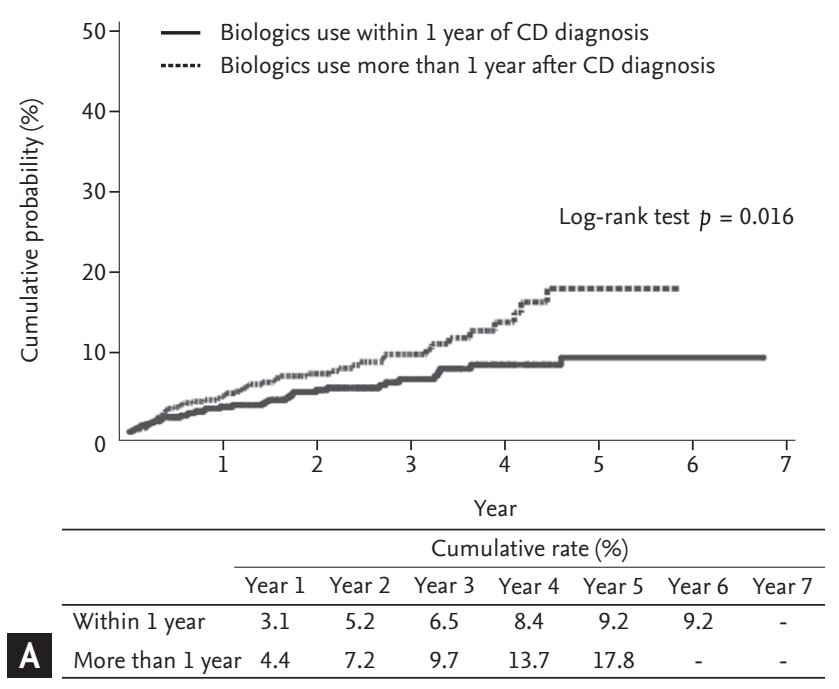

Hospitalization

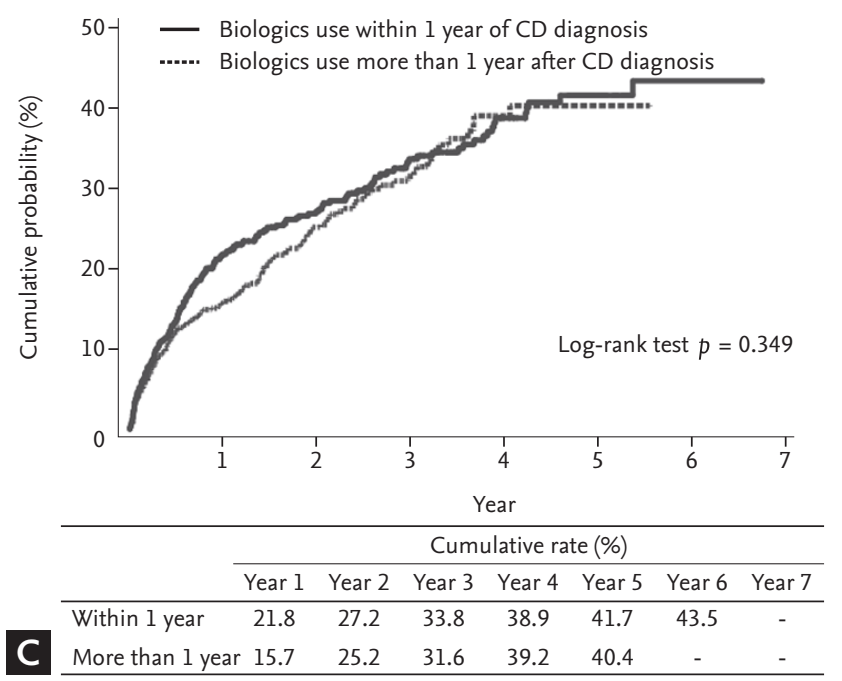

Emergency room visit



Emergency room visit

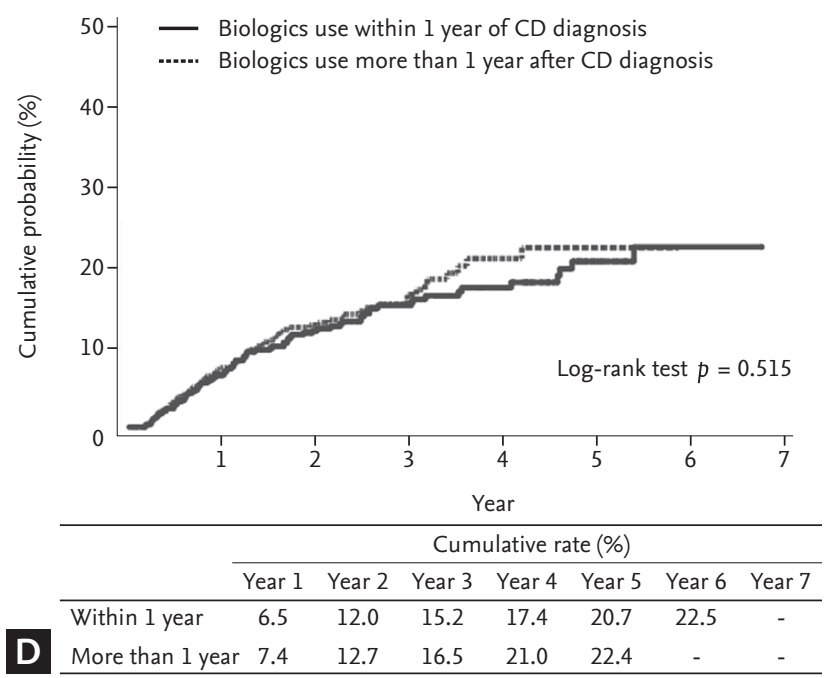

Figure 2. Kaplan-Meier survival analysis for clinical outcomes of anti-tumor necrosis factor therapy. Cumulative probabilities of (A) abdominal surgery, (B) emergency room visit, (C) hospitalization, and (D) new corticosteroid use. CD, Crohn's disease.

surgery before the start of anti-TNF therapy. Ultimately, 1,207 patients were included in the study, of whom 609 were early initiators of anti-TNF ( $\leq 1$ year) and 598 were late initiators of anti-TNF ( $>1$ year) therapy (Fig. 1). The median period from CD diagnosis to anti-TNF initiation in all 1,207 patients was 1.0 years (interquartile range [IQR], 0.4 to 2.2). Among the 609 early initiators, $410(67.3 \%)$ started with infliximab and 199 (32.7\%) started with adalimumab. Among the 598 late initiators,
305 (51.0\%) and 293 (49.0\%) started with infliximab and adalimumab, respectively.

Table 1 describes the baseline characteristics of early and late initiators of anti-TNF therapy. Sex and age at diagnosis of CD were similar between the two groups. The age at the time of first anti-TNF use was lower in early initiators than in late initiators, and the mean period of anti-TNF use was longer in early initiators than in late initiators. The rates of 5-ASAs and steroid use at 
Table 1. Baseline characteristics of study population

\begin{tabular}{|c|c|c|c|}
\hline Characteristic & $\begin{array}{l}\text { Early initiators of anti-TNF } \\
\qquad(\mathrm{n}=609)\end{array}$ & $\begin{array}{l}\text { Late initiators of anti-TNF } \\
\qquad(\mathrm{n}=598)\end{array}$ & $p$ value \\
\hline Male sex & $422(69 \cdot 3)$ & $431(72.1)$ & 0.319 \\
\hline Age at diagnosis of CD, yr & $24.8 \pm 11.3$ & $24.9 \pm 10.7$ & 0.925 \\
\hline$<30$ & $473(77 \cdot 7)$ & $451(75 \cdot 4)$ & 0.393 \\
\hline$\geq 30$ & $136(22.3)$ & $147(24.6)$ & \\
\hline Age at the start of anti-TNF & $25.2 \pm 11.3$ & $27.4 \pm 10.7$ & $<0.001$ \\
\hline The period from CD diagnosis to first anti-TNF use, yr & $0.4 \pm 0.3$ & $2.5 \pm 1.2$ & $<0.001$ \\
\hline Anti-TNF use period, yr & $2.5 \pm 1.6$ & $2.2 \pm 1.3$ & $<0.001$ \\
\hline \multicolumn{4}{|l|}{ First anti-TNF agent } \\
\hline Infliximab & $410(67 \cdot 3)$ & $305(51.0)$ & $<0.001$ \\
\hline Adalimumab & $199(32.7)$ & $293(49.0)$ & \\
\hline \multicolumn{4}{|l|}{ Medication use at first anti-TNF use } \\
\hline 5-ASAs & $379(62.2)$ & $334(55 \cdot 9)$ & 0.028 \\
\hline Steroids & $173(28.4)$ & $107(17.9)$ & $<0.001$ \\
\hline Immunomodulators & $344(56.5)$ & $323(54 \cdot 0)$ & 0.420 \\
\hline Concomitant immunomodulators (+/-30 days) & $462(75 \cdot 9)$ & $447(74.8)$ & 0.703 \\
\hline Previous immunomodulators exposure & $518(85.1)$ & $549(91.8)$ & $<0.001$ \\
\hline \multicolumn{4}{|l|}{ Region at first anti-TNF use } \\
\hline Seoul & $251(41.2)$ & $310(51.8)$ & $<0.001$ \\
\hline Outside Seoul & $358(58.8)$ & $288(48.2)$ & \\
\hline \multicolumn{4}{|l|}{ Hospital scale at first anti-TNF use } \\
\hline Tertiary hospitals & $420(69.0)$ & $458(76.6)$ & 0.004 \\
\hline General hospitals/community hospitals/clinics & $189(31.0)$ & $140(23.4)$ & \\
\hline Surgical history of perianal disease before anti-TNF use ${ }^{a}$ & $22(3.6)$ & $41(6.9)$ & 0.016 \\
\hline
\end{tabular}

Values are presented as number (\%) or mean \pm SD.

TNF, tumor necrosis factor; CD, Crohn's disease; 5-ASA, 5-aminosalicylic acid.

${ }^{a}$ Surgical history of perianal disease was defined as the presence of surgical procedure codes for anal fissure (Q2950), anal fistula (Q2974, Q2975, Q2976 Q2977, Q2978, and Q2979), or periproctal abscess (Q2881, Q2882, and Q2883) before starting biological therapy.

first anti-TNF use were higher in early initiators than in late initiators, while the rate of concomitant IM use was not different between the two groups. The proportion of patients who first started anti-TNF therapy in the Seoul region and at tertiary hospitals was lower among early initiators than among late initiators. Surgical history of perianal disease before anti-TNF use was higher in late initiators than in early initiators (6.9\% vs. 3.6\%, $p=0.016)$.

\section{Comparison of clinical outcomes between early and late initiators of anti-TNF}

Late initiators of anti-TNF showed significantly higher cumulative probabilities of abdominal surgery $(p=$
0.016) (Fig. 2A) and CD-related ER visit $(p=0.036)$ (Fig. 2B) than early initiators of anti-TNF. However, there were no significant differences in the cumulative probabilities of CD-related hospitalization $(p=0.349)$ (Fig. $2 \mathrm{C}$ ) and corticosteroid use $(p=0.515)$ (Fig. 2D) between early and late initiators. At 1, 3, and 5 years after starting the first anti-TNF agent, cumulative rates of abdominal surgery were $3.1 \%, 6.5 \%$, and $9.2 \%$, respectively, among early initiators, and 4.4\%, 9.7\%, and $17.8 \%$, respectively, among late initiators.

Median time to surgery, ER visit, hospitalization, and corticosteroid use after anti-TNF initiation was similar between early and late initiators (Table 2). 
Table 2. Comparison of outcomes between early and late initiators of anti-TNF agent

\begin{tabular}{|c|c|c|c|}
\hline Variable & $\begin{array}{l}\text { Early initiators of anti-TNF } \\
\qquad(\mathrm{n}=6 \circ 9)\end{array}$ & $\begin{array}{l}\text { Late initiators of anti-TNF } \\
\qquad(\mathrm{n}=598)\end{array}$ & $p$ value \\
\hline \multicolumn{4}{|l|}{ Abdominal surgery outcomes } \\
\hline Surgery, n (\%) & $36(5 \cdot 9)$ & $51(8.5)$ & 0.099 \\
\hline Surgery rate, /100 patient-years, 95\% CI & $2.2(1.6-3.0)$ & $3.9(2.9-5.1)$ & 0.008 \\
\hline Time to surgery, yr, median (IQR) & $1.03(0.34-2.05)$ & $1.00(0.38-2.23)$ & 0.983 \\
\hline \multicolumn{4}{|l|}{ ER visit outcomes } \\
\hline ER visit, $\mathrm{n}(\%)$ & $70(11.5)$ & $78(13.0)$ & 0.464 \\
\hline ER visit rate, /100 patient-years, $95 \% \mathrm{CI}$ & $4 \cdot 2(3 \cdot 3-5 \cdot 3)$ & $5.8(4.6-7.2)$ & 0.045 \\
\hline Time to ER visit, yr, median (IQR) & $1.75(0.43-2.91)$ & $1.42(0.67-2.77)$ & 0.721 \\
\hline \multicolumn{4}{|l|}{ Hospitalization outcomes } \\
\hline Hospitalization, $\mathrm{n}(\%)$ & $186(30.5)$ & $155(25 \cdot 9)$ & 0.086 \\
\hline Hospitalization rate, /100 patient-years, 95\% CI & $14.5(12.5-16.6)$ & $14.2(12.1-16.5)$ & 0.850 \\
\hline Time to Hospitalization, yr, median (IQR) & $0.57(0.19-1.38)$ & $0.60(0.19-1.66)$ & 0.761 \\
\hline \multicolumn{4}{|l|}{ New steroid use (after 2 months) outcomes } \\
\hline New steroid use, $\mathrm{n}(\%)$ & $82(13 \cdot 5)$ & $79(13.2)$ & 0.964 \\
\hline New steroid use rate, /100 patient-years, 95\% CI & $5 \cdot 4(4 \cdot 3-6.6)$ & $6.3(5.0-7.8)$ & 0.292 \\
\hline Time to new steroid use, yr, median (IQR) & $1.07(0.59-1.96)$ & $0.93(0.49-1.60)$ & 0.480 \\
\hline
\end{tabular}

TNF, tumor necrosis factor; CI, confidence interval; IQR, interquartile range.

\section{Cox proportional hazard regression analysis for factors associated with clinical outcomes}

The HRs in univariate and multivariable Cox proportional hazards models are summarized in Table 3. Univariate analysis showed that late initiation of anti-TNF therapy was associated with increased risks of surgery (HR, 1.68; 95\% CI, 1.10 to 2.59) and ER visit (HR, 1.42; 95\% CI, 1.02 to 1.96). Even after adjustment for confounding variables, late initiation of anti-TNF therapy was independently associated with a higher risk of surgery (adjusted hazard ratio [aHR], 1.64; 95\% CI, 1.05 to 2.55) and tended to be associated with a higher risk of ER visit, although this result was not statistically significant (aHR, 1.38; 95\% CI, 0.99 to 1.94). However, there were no significant differences in the risk of hospitalization and corticosteroid use between early and late initiators.

In multivariable Cox regression analysis, several other factors were also identified as being associated with clinical outcomes. Age at the start of anti-TNF therapy was associated with increased risk of surgery (aHR, 1.02; 95\% CI, 1.01 to 1.04), hospitalization (aHR, 1.01; 95\% CI, 1.01 to 1.02), and corticosteroid use (aHR, 1.02; 95\% CI, 1.01 to 1.03). The anti-TNF use period (/years; aHR, o.81; $95 \% \mathrm{CI}, 0.69$ to 0.95 ) and initiation of anti-TNF in the
Seoul region (aHR, 0.73; 95\% CI, 0.58 to 0.93 ) were associated with a reduced risk of hospitalization. The use of adalimumab initiators was associated with a higher risk of ER visits than that of infliximab initiators (aHR, 1.85; 95\% CI, 1.32 to 2.59 ).

Because previous studies have proposed a cut-off time of 2 years for early CD treatment [8-11], we conducted further analysis to assess the effect of initiation of anti-TNF therapy within 2 years after diagnosis. Of 1,207 patients, 873 started anti-TNF therapy within 2 years of diagnosis, and 334 started anti-TNF therapy $>2$ years after diagnosis. In multivariable Cox regression analysis, late initiation of anti-TNF therapy $>2$ years after diagnosis was independently associated with higher risk of ER visit (aHR, 1.65; 95\% CI, 1.13 to 2.41). However, there were no significant differences in terms of the risk of surgery, hospitalization, and corticosteroid use between early ( $\leq 2$ year) and late initiators (> 2 years) (Supplementary Table 1).

\section{DISCUSSION}

In this study, using a large population-level claims database, we were able to clearly identify an association 


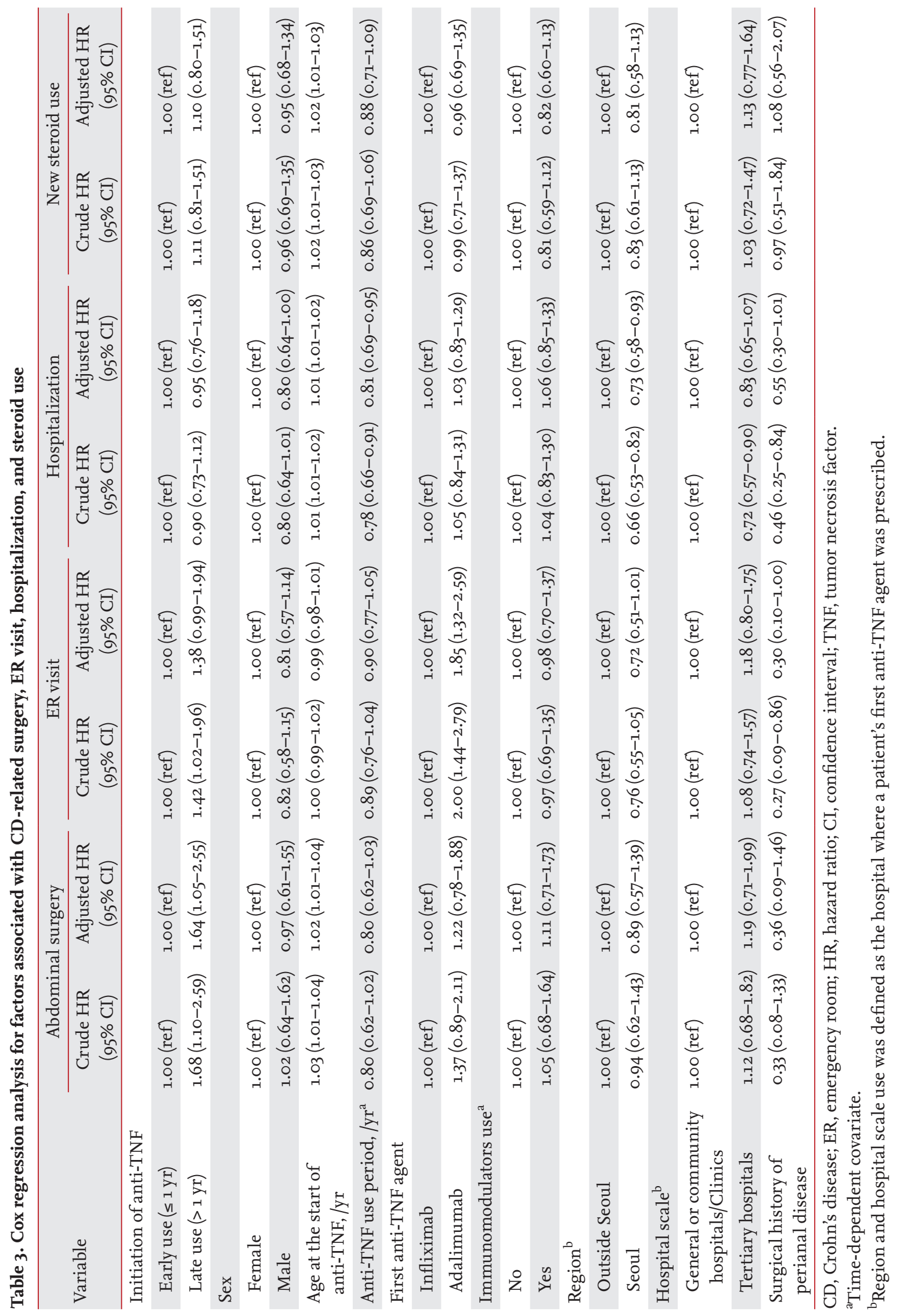


between early anti-TNF initiation and several beneficial clinical outcomes in CD. More specifically, early treatment with anti-TNF agents within 1 year of diagnosis was independently associated with reduced risk of abdominal surgery and tended to be associated with reduced risk of CD-related ER visit.

To date, several studies have explored the impact of anti-TNF treatment timing on clinical outcomes in patients with $\mathrm{CD}$, although the definitions of early anti-TNF initiation and clinical outcomes investigated vary slightly among studies. Similar to our results, most previous studies have shown more favorable outcomes in early initiators than in late initiators. A retrospective cohort study using the MarketScan Commercial and Medicare databases in the United States reported a negative association between lagged use of biologics and the proportion of patients with IBD-related hospitalizations and ER visits [15]. In this study, biologic use in the previous year was associated with a $3.8 \%$ to $5.6 \%$ reduction in hospitalizations in the current year, and similar results were observed for ER visits [15]. However, this study considered patients with both ulcerative colitis (UC) and CD together in a single category despite the fact that these two diseases can manifest quite differently. A Spanish study revealed that time between diagnosis and anti-TNF initiation was associated with the risk of major abdominal surgery in CD [16]. More specifically, out of 272 patients who received anti-TNF therapy, 137 patients who had undergone surgery started anti-TNF agents after a median of 166 months (IQR, 90 to 233) after diagnosis, while patients who had not undergone surgery started therapy after a median of 59 months (IQR, 14 to $162 ; p<0,005)[16]$. The odds ratio for surgery was 1.008 (95\% CI, 1.005 to 1.010) for each month of delay in starting anti-TNFs [16]. A Canadian retrospective study involving $190 \mathrm{CD}$ patients (100 receiving infliximab and 90 receiving adalimumab) demonstrated that early initiation of anti-TNF therapy within the first 2 years of diagnosis reduced the rate of surgery and secondary loss of response requiring dose escalation [8]. Additionally, the Swiss IBD Cohort Study (SIBDCS) involving 540 CD patients (the data obtained from patients enrolled in SIBDCS between 2006 and 2012) found that treatment with anti-TNF agents within the first 2 years of CD diagnosis was associated with reduced risk of developing bowel strictures, when compared with initiating these drugs $>2$ years after diagnosis $(\mathrm{HR}, 0.276 ; p=0.018)$ [9]. Recently, the data obtained from SIBDCS involving more patients (246 early initiators and 696 late initiators) over a longer period of time (between 2006 and 2016) also showed that early anti-TNF administration within 2 years of CD diagnosis was associated with reduced risk of osteoporosis and anemia as well as bowel stenosis [10]. Furthermore, patients with early anti-TNF administration sought medical consultations significantly less frequently (including gastroenterologists in private practice, outpatient hospital visits, and a composite of any medical visits) and, by trend, showed lower rates of absence from work owing to $\mathrm{CD}$, in contrast to patients with late anti-TNF administration [10]. In line with these results, a South Korean study reported that late anti-TNF/IMs treatment (initiating these drugs $>2$ years after diagnosis) was independently associated with higher risks of intestinal surgery (aHR, 2.32), behavioral progression (aHR, 2.00), stricturing complications (aHR, 1.74), and penetrating complications (aHR, 3.32) in patients with CD [11]. However, this study was based on the data obtained from a single tertiary referral center and included only 79 early anti-TNF initiators and did not analyze these patients separately.

In contrast to the results of our study and previous studies, a population-based study in the Netherlands did not find a beneficial effect of starting anti-TNF therapy early (<16 months) in contrast to starting anti-TNF therapy late (> 16 months) with respect to surgery, abscess formation, fistula formation, extra-intestinal manifestations (EIMs), and mucosal healing [17]. However, the number of patients included in this study was too small to draw a definitive conclusion; only $66 \mathrm{CD}$ patients and 16 UC patients were included, and these two diseases were analyzed together.

The existing literature and our findings support that the early use of anti-TNF may alter the natural history of CD. Early anti-TNF therapy may rapidly reduce intestinal inflammation before the onset of permanent scar formation, thereby inhibiting the development of mechanical complications such as fibrostenotic stricturing and penetrating disease. The reduction in these irreversible complications in the early stages of disease may have led to a decrease in the rate of surgeries. However, the cost effectiveness of early anti-TNF initiation is a matter of concern. Although early initiation of anti-TNF 
agents can reduce the rates of CD-related complications, surgery, or ER visit, these drugs are very expensive. Therefore, the longer-term use of anti-TNF agents may cause economic burden. Indeed, some studies since the advent of biologic agents have reported that increased use of anti-TNF agents has been the key driver of CD-related healthcare costs $[18,19]$. Recently, a Korean population-based study also demonstrated that anti-TNF agents accounted for $93.1 \%$ of all medication costs and $68.8 \%$ of the total cost in 2015 [20]. However, a Canadian study found that early initiation of anti-TNF therapy (both infliximab and adalimumab) within 2 years of CD diagnosis provides significant cost savings and quality-adjusted life-years compared with later initiation [21]. This study suggests that early anti-TNF therapy has the potential not only to improve clinical outcomes, but also to reduce the economic burden associated with $\mathrm{CD}$ [21]. The cost effectiveness of early initiation of anti-TNF therapy may be different between countries, because the medical environments vary among different countries and the effect of anti-TNF therapy may vary from race to race. Thus, more worldwide studies are needed to verify the cost effectiveness of early initiation of anti-TNF therapy. Another concern with early anti-TNF therapy is safety, especially in terms of the risk of lymphoma and infections. Further large-scale, long-term studies are warranted to assess the safety of early anti-TNF therapy.

Interestingly, we found that age at the start of anti-TNF was associated with higher risks of surgery, hospitalization, and corticosteroid use. In other words, among CD patients who received anti-TNF therapy for more than 6 months, older age at the start of anti-TNF was associated worse clinical outcomes. This may be because anti-TNF agents are more effective in patients with high inflammatory burden and no irreversible changes in the intestine. Younger patients usually have more inflammatory burden and less irreversible changes in the intestine than older patients. Similar to our findings, a multi-national, multicenter study revealed that younger age at the start of infliximab therapy was associated with a beneficial long-term use [22].

To the best of our knowledge, this is the first population-based study to assess the impact of early initiation of anti-TNF on clinical outcomes of CD patients in Asia. However, the present study has some limitations. First, disease activity such as clinical, biochemical, or endo- scopic activity, phenotypes such as the location and behavior of CD, and indication of anti-TNF use which can affect clinical outcomes, were not considered because the administrative data do not include these details. However, since infliximab and adalimumab were approved only for patients with a CD activity index of 220 or higher in South Korea, the clinical activity of patients included in this study may not have been significantly different. Second, although several clinical outcomes including surgery were analyzed, more detailed clinical outcomes, such as intestinal complications (including stenosis, fistula, and abscess), loss of response requiring dose escalation of anti-TNF agent, and EIMs, were not examined. Third, we were unable to assess the efficacy of early anti-TNF therapy for perianal disease because the claims codes could not distinguish perianal disease. However, we considered the surgical history of perianal diseases and this factor was adjusted in our analysis. Fourth, the definition of CD-related ER visits may not be perfect. ER visits with $\mathrm{CD}$ as the primary diagnosis may be difficult to accurately regard as CD-related ER visits. Lastly, we did not evaluate the cost effectiveness and safety of early initiation of anti-TNF therapy.

Despite these limitations, our study provides a better understanding of the impact of the early use of anti-TNF agents on clinical outcomes in patients with CD. In conclusion, an association between early anti-TNF therapy within 1 year of diagnosis and better clinical outcomes, including reduced risks of abdominal surgery and ER visit, was observed in Korean patients with CD. Our findings provide evidence supporting that aggressive medical intervention in the early stages of CD can potentially change the natural history of the disease in relation to surgery and clinical outcomes.

\section{KEY MESSAGE}

1. Early treatment with anti-tumor necrosis factor agents within 1 year of diagnosis was associated with reduced risk of abdominal surgery and tended to be associated with reduced risk of Crohn's disease (CD)-related emergency room visit.

2. These results suggest that aggressive medical intervention in the early stages of CD may potentially change the course of this disease. 


\section{Conflict of interest}

No potential conflict of interest relevant to this article was reported.

\section{REFERENCES}

1. Lee SH, Kwon JE, Cho ML. Immunological pathogenesis of inflammatory bowel disease. Intest Res 2018;16:26-42.

2. Ooi CJ, Hilmi I, Banerjee R, et al. Best practices on immunomodulators and biologic agents for ulcerative colitis and Crohn's disease in Asia. Intest Res 2019;17:285-310.

3. Im JP, Ye BD, Kim YS, Kim JS. Changing treatment paradigms for the management of inflammatory bowel disease. Korean J Intern Med 2018;33:28-35.

4. Lichtenstein GR, Yan S, Bala M, Blank M, Sands BE. Infliximab maintenance treatment reduces hospitalizations, surgeries, and procedures in fistulizing Crohn's disease. Gastroenterology 2005;128:862-869.

5. Feagan BG, Panaccione R, Sandborn WJ, et al. Effects of adalimumab therapy on incidence of hospitalization and surgery in Crohn's disease: results from the CHARM study. Gastroenterology 2008;135:1493-1499.

6. Rubin DT, Uluscu O, Sederman R. Response to biologic therapy in Crohn's disease is improved with early treatment: an analysis of health claims data. Inflamm Bowel Dis 2012;18:2225-2231.

7. Khanna R, Bressler B, Levesque BG, et al. Early combined immunosuppression for the management of Crohn's disease (REACT): a cluster randomised controlled trial. Lancet 2015;386:1825-1834.

8. Ma C, Beilman CL, Huang VW, et al. Anti-TNF therapy within 2 years of Crohn's disease diagnosis improves patient outcomes: a retrospective cohort study. Inflamm Bowel Dis 2016;22:870-879.

9. Safroneeva E, Vavricka SR, Fournier N, et al. Impact of the early use of immunomodulators or TNF antagonists on bowel damage and surgery in Crohn's disease. Aliment Pharmacol Ther 2015;42:977-989.

10. Frei R, Fournier N, Zeitz J, et al. Early initiation of anti-TNF is associated with favourable long-term outcome in Crohn's disease: 10-year-follow-up data from the Swiss IBD cohort study. J Crohns Colitis 2019;13:1292-1301.

11. Oh EH, Oh K, Han M, et al. Early anti-TNF/immunomodulator therapy is associated with better long-term clinical outcomes in Asian patients with Crohn's disease with poor prognostic factors. PLoS One 2017;12:e0177479.

12. Jung YS, Han M, Kim WH, Park S, Cheon JH. Incidence and clinical outcomes of inflammatory bowel disease in South Korea, 2011-2014: a nationwide population-based study. Dig Dis Sci 2017;62:2102-2112.

13. Jung YS, Han M, Park S, Kim WH, Cheon JH. Cancer risk in the early stages of inflammatory bowel disease in Korean patients: a nationwide population-based study. J Crohns Colitis 2017;11:954-962.

14. Han M, Jung YS, Cheon JH, Park S. Regional variations in the use of biologics and immunomodulators among Korean patients with inflammatory bowel diseases. J Gastroenterol Hepatol 2019;34:1166-1174.

15. David G, Gunnarsson C, Lofland JH. Variations in care: a retrospective database analysis of healthcare utilization patterns for patients with inflammatory bowel disease. J Med Econ 2015;18:137-144.

16. Gonzalez-Lama Y, Suarez C, Gonzalez-Partida I, et al. Timing of thiopurine or anti-TNF initiation is associated with the risk of major abdominal surgery in Crohn's disease: a retrospective cohort study. J Crohns Colitis 2016;10:55-6o.

17. Nuij V, Fuhler GM, Edel AJ, et al. Benefit of earlier anti-TNF treatment on IBD disease complications? J Crohns Colitis 2015;9:997-1003.

18. Park KT, Colletti RB, Rubin DT, Sharma BK, Thompson A, Krueger A. Health insurance paid costs and drivers of costs for patients with Crohn's disease in the United States. Am J Gastroenterol 2016;111:15-23.

19. van der Valk ME, Mangen MJ, Leenders M, et al. Healthcare costs of inflammatory bowel disease have shifted from hospitalisation and surgery towards anti-TNF $\alpha$ therapy: results from the COIN study. Gut 2014;63:72-79.

20. Kim JW, Lee CK, Lee JK, et al. Long-term evolution of direct healthcare costs for inflammatory bowel diseases: a population-based study (2006-2015). Scand J Gastroenterol 2019;54:419-426.

21. Beilman CL, Kirwin E, Ma C, McCabe C, Fedorak RN, Halloran B. Early initiation of tumor necrosis factor antagonist-based therapy for patients with Crohn's disease reduces costs compared with late initiation. Clin Gastroenterol Hepatol 2019;17:1515-1524.

22. Juillerat $\mathrm{P}$, Sokol H, Froehlich F, et al. Factors associated with durable response to infliximab in Crohn's disease 5 years and beyond: a multicenter international cohort. Inflamm Bowel Dis 2015;21:60-70. 


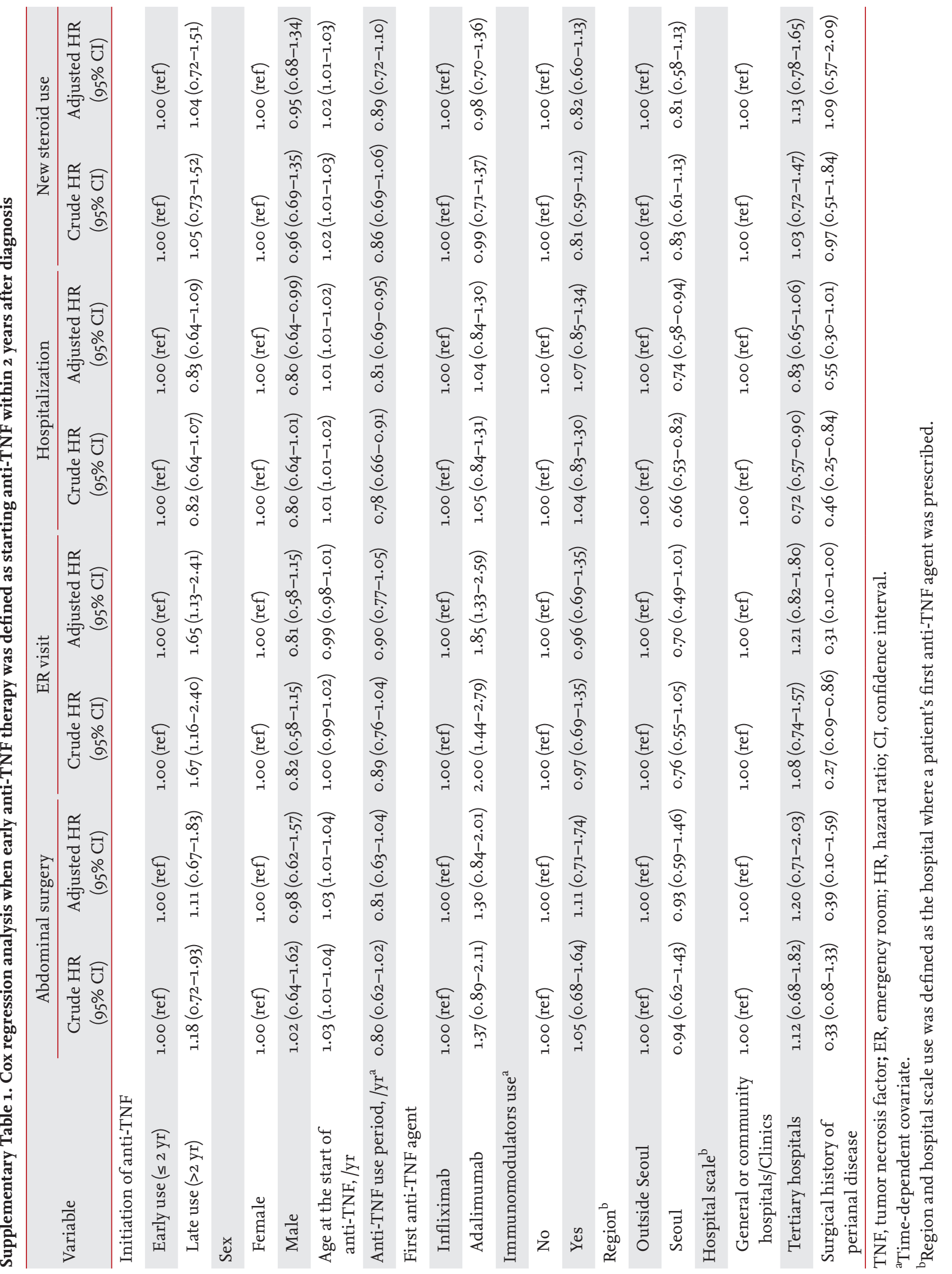

